\title{
Les narrations brèves dans un long roman, Artamene ou le Grand Cyrus des Scudéry
}

\section{Marie-Gabrielle Lallemand}

\section{OpenEdition}

12 Journals

Édition électronique

URL : https://journals.openedition.org/rhetorique/1288

DOI : $10.4000 /$ rhetorique. 1288

ISSN : 2270-6909

Éditeur

UGA Éditions/Université Grenoble Alpes

Édition imprimée

ISBN : 978-2-37747-345-8

Référence électronique

Marie-Gabrielle Lallemand, "Les narrations brèves dans un long roman, Artamene ou le Grand Cyrus des Scudéry », Exercices de rhétorique [En ligne], 18| 2022, mis en ligne le 22 février 2022, consulté le 26 février 2022. URL : http://journals.openedition.org/rhetorique/1288 ; DOI : https://doi.org/10.4000/ rhetorique. 1288

Ce document a été généré automatiquement le 26 février 2022.

\section{(c) (7) ()}

Les contenus de la revue Exercices de rhétorique sont mis à disposition selon les termes de la Licence Creative Commons Attribution - Pas d'Utilisation Commerciale - Partage dans les Mêmes Conditions 4.0 International. 


\title{
Les narrations brèves dans un long roman, Artamene ou le Grand Cyrus des Scudéry
}

\author{
Marie-Gabrielle Lallemand
}

1 S'intéresser à la brièveté dans un long roman peut sembler paradoxal. Mais si le long roman, qu'il relève de la veine pastorale ou de la veine héroïque, est long, c'est qu'il recèle une multitude de récits insérés dans un récit cadre, et que ces récits sont comparativement plus courts, voire brefs. Autrement dit, coexistent dans le long roman un récit qui s'étend sur l'ensemble des tomes du roman, qui est le récit principal, et des récits qui n'en constituent qu'une petite partie voire une toute petite partie et qui sont enchâssés dans le récit premier ${ }^{1}$. Si plus particulièrement, c'est Artamene ou le Grand Cyrus des Scudéry (1649-1653, dix volumes, 7840 pages dans l'édition Barbin de 1656) qui a retenu notre attention, c'est que d'emblée, dès le début de ce roman, deux modalités de narration, l'une longue, l'autre brève sont envisagées. La première, le récit circonstancié, est réalisée : il s'agit de la relation de la vie de Cyrus par deux de ses fidèles serviteurs à un groupe de princes (« Histoire d'Artamene », « Suite de l'histoire d'Artamene »). La seconde, le récit sommaire, n'est qu'évoquée : il s'agit de cette même relation faite de nouveau à un prince qui n'a pu entendre le premier récit. La question de la longueur de la narration est donc posée et c'est la narration longue qui est préférée, même si la narration brève ne manque pas d'efficacité : « quoy que ce ne fust qu'un simple abregé de la vie d'Artamene», il «charma » son auditeur et «redoubla encore l'estime » qu'il avait pour Artamène ${ }^{2}$.

2 Une autre raison nous a amenée à faire le choix de ce roman : le premier récit inséré est explicitement une narration judiciaire. Artamène, accusé d'intelligence avec l'ennemi, est emprisonné et sa vie est menacée. Ses fidèles serviteurs se décident alors à faire connaître son identité et à raconter sa vie à un aréopage de princes qui peuvent intervenir en sa faveur. Il s'agit par le récit de faire connaître quel est l'homme qu'on accuse, pour convaincre qu'un tel homme ne peut avoir commis le crime qu'on lui impute $^{3}$. Au terme de la narration, les auditeurs-juges sont convaincus de l'innocence 
d'Artamène qu'ils connaissent désormais pour être Cyrus. Certes, il ne s'agit pas stricto sensu d'un procès et les auditeurs ne vont pas prononcer un jugement en bonne et due forme mais, dans le passage en question, le vocabulaire ayant trait à la justice abonde ${ }^{4}$, signalant qu'un modèle narratif sous-jacent dans le récit de la vie d'Artamène est celui de la narration judiciaire.

Artamène ou le Grand Cyrus se présente donc comme un bon terrain d'étude du rapport entre narratio et narration.

\section{Les narrations judiciaires}

4 Les narrations insérées dans le récit premier sont introduites en sorte que leurs visées soient déterminées avant qu'elles ne débutent. Cela sert à donner de l'unité au récit. Les nombreuses histoires qui se greffent sur le récit principal sont ainsi rendues nécessaires par le contexte: elles ont une raison d'être. Cette visée ancre parfois sensiblement les narrations dans un genre rhétorique. C'est le cas, par exemple, de la narration que fait Philoclès de l'« Histoire de Cléobuline, reine de Corinthe » à Cyrus afin qu'il intervienne pour persuader la reine de prendre un époux ${ }^{5}$. Ici, la narration s'inscrit dans un cadre délibératif. Ailleurs, et ce qui va maintenant nous intéresser, le cadre est judiciaire. La narration est alors une des parties d'une séquence: elle est précédée d'une accusation ou d'une demande de jugement d'un différend, elle se poursuit par une confirmation et s'achève par un jugement. Pour ces narrations judiciaires, le modèle des tribunaux d'amour que l'on trouve dans l'Astrée est prégnant, tribunaux où sont jugées des "questions galantes ${ }^{6}$ ». Dans le cas des «Histoires des Amants infortunez ", la séquence est d'un seul tenant. Une assemblée qui s'est réunie chez Martésie discute des maux d'amour. Quatre princes déclarent qu'ils souffrent les pires maux : ils parlent d'expérience, affirment-ils, avant d'exposer l'un après l'autre quelques-unes de leurs « raisons ${ }^{7}$ ». Le différend ne peut être tranché que par un juge qui «ne soit pas preocupé en sa propre cause »: ce sera Martésie ${ }^{8}$. Les quatre princes racontent chacun leur histoire. Le premier, le deuxième et le quatrième récit sont composés d'un exorde, d'une narration et d'une confirmation. Le deuxième narrateur fait l'impasse sur l'exorde. À l'issue des narrations, après consultation de l'assemblée, un jugement est prononcé. L'organisation est précisée avant que ne commence les récits. L'un des devisants, s'adressant à Martésie, lui déclare en effet :

[...] il faut qu'apres avoir escouté le recit de nos avantures, \& en suite nos raisons ; vous jugiez souverainement, lequel est le plus malheureux, ou de celuy qui est presque toujours absent de ce qu'il aime : ou de celuy qui n'est point aimé ; ou de celuy qui a veû mourir la personne aimée : ou de celuy qui est effroyablemnt jaloux ${ }^{9}$ [...].

5 C'est encore une séquence d'un seul tenant qui contient l'« Histoire de Thrasyle ${ }^{10}$ ». Thrasyle est accusé d'inconstance, et il s'en défend. Dans ce temps d'accusation, on retrouve du vocabulaire juridique ${ }^{11}$. Suit la narration mais afin que, par ce récit, on puisse juger en bonne connaissance de cause, Thrasyle est récusé comme narrateur car il trop impliqué dans l'affaire. La narratrice choisie devra se montrer neutre et s'y engage $^{12}$. La narration est suivie du jugement, directement ${ }^{13}$, sans qu'il y ait de confirmation: les auditeurs refusent de conseiller la juge, de sorte que les arguments en faveur ou en défaveur de Thrasyle ne sont pas donnés. En revanche, dans un autre tribunal d'amour, une confirmation suit les récits du personnage qui soutient que l'on peut aimer deux fois la même personne, dans l'«Histoire de Philidaspe et 
d'Anaxandrine ${ }^{14}$ », et du personnage qui soutient le contraire, dans l'« Histoire d'Aglatonice et d'Iphicrate ${ }^{15} »$, avant que ne soit prononcé un jugement en faveur du premier narrateur, après consultation de l'assemblée ${ }^{16}$. On trouve, à une reprise, une autre dispositio, qui éloigne le jugement de la narration. L'« Histoire d'Arpalice et de Thrasimède ${ }^{17}$ ", est entendue par Cyrus qui veut connaître la "cause de [la] querelle " qui oppose Thrasimède et Ménécrate, afin de "pouvoir terminer le «different » qui oppose les deux hommes ${ }^{18}$. De nouveau, on observe la présence de vocabulaire relevant du judicaire ("juger», "tesmoigner», " accuser injustement $\left.{ }^{19} »\right)$ qui signale que le modèle de récit est celui de la narratio judiciaire. C'est finalement à Doralise, qui a l'oreille d'Andramite, un proche de Cyrus, que le récit des aventures des deux rivaux et de leur maîtresse va être fait. Au terme du récit, Doralise demande à Andramite d'intervenir en faveur de Thrasimède auprès de Cyrus. Mais ce n'est que dans la partie suivante que la confirmation et le jugement vont trouver place, et non immédiatement après la narratio. Sont alors rapportés les arguments des partis en présence, avant que Cyrus ne consulte son entourage et qu'ensemble ils débattent de la cause, avant qu'un jugement unanime en faveur de Trasimède ne soit rendu ${ }^{20}$.

6 Les narrations à visée judiciaire ne sont pas les mieux représentées dans ce roman, comme dans les romans du temps, loin de là. Dans Artamene, en règle générale, les visées sont plurielles et la narration, quand elle a une visée judiciaire, n'est qu'entre autres un plaidoyer. Nous prendrons l'« Histoire de Sésostris et de Timarete» comme exemple. Un chef égyptien, Miris, s'oppose à Cyrus qui veut secourir un homme dont il a admiré la vaillance, mais, dit Miris, il s'agit d'« un assassinateur de Rois [...], un homme que l'amour \& l'ambition ont noircy de tous les crimes imaginables ${ }^{21}$ ». Cet homme, de plus, est le rival du prince Sésostris, maître de Miris, et il détient une information capitale pour ce prince. À la demande de ce dernier, Miris se propose de raconter à Cyrus leur histoire "afin que vous puissiez sçavoir combien il importe à Sesostris, de n'ignorer pas ce qu'Heracleon seulement peut luy apprendre : \& que vous sçachiez aussi, quelle différence vous devez faire entre ces deux Rivaux ${ }^{22} »$. Cyrus lui répond favorablement : Miris lui fera un "plaisir signalé », parce que depuis qu'il a rencontré Sésostris, il a «toujours eu envie de le connoitre un peu plus qu'[il] ne fai $[\mathrm{t}]^{23} »$.

7 Il s'agit bien, on le voit, de prouver par la narration la culpabilité d'Héracléon, mais il s'agit aussi par ce récit de faire en sorte que Cyrus aide Sésostris à obtenir une information importante. Cyrus, quant à lui, est curieux de mieux connaître la vie d'un prince admirable, raison pour laquelle il demande à Miris de « luy dire bien exactement toute la vie de Sesostris ${ }^{24} »$. Nous sera donc livré un récit long de 205 pages.

\section{De la longueur de la narration}

Dans Artamene ou le Grand Cyrus, le choix narratif du bref ou du long est régulièrement explicite. Ainsi, Philoclès qui, comme on l'a vu, va livrer un récit plutôt bref (une centaine de pages) à visée délibérative, commence par faire savoir à son auditeur que, s'écartant de l'usage, il ne racontera pas l'histoire de la race de Cléobuline ${ }^{25}$ ni même l'enfance de celle-ci, mais débutera son récit au moment où Cléobuline accède au trône ${ }^{26}$. On observe que, conformément aux préconisations de la rhétorique, plus la visée judiciaire du récit est manifeste plus les récits sont brefs, de sorte que les récits les plus courts sont ceux qui interviennent dans les tribunaux $d^{\prime} a m o u r{ }^{27}$. Cette brièveté 
entraîne un commentaire d'Eumétis, une auditrice qui déclare avant que ne commence la relation d'une histoire "qu'elle seroit bien aise qu'un Grec Asiatique exagerast un peu plus les choses» que le précédent narrateur qui avait donné un «récit en Lacédémonien ${ }^{28}$ ». Il n'en demeure pas moins que le deuxième récit (56 pages), s'il est plus développé que le premier (16 pages), est comparativement aux autres récits du roman bref.

Pour quelles raisons faire le choix d'une narration brève ? Philoclès, un des amants infortunés, déclare qu'il convient quand on ne peut maintenir le lecteur dans l'incertitude du dénouement :

Comme vous sçavez la fin de mon avanture, auparavant que d'en avoir apris le commencement ny la suitte : \& que par consequent cette agreable suspension, qui fait que l'on escoute quelquefois les choses fascheuses avec plaisir, ne se peut trouver dans mon recit; je pense qu'il est à propos de n'abuser pas de vostre patience, par une narration extrémement estenduë. Je vous diray donc seulement ${ }^{29}$ [...].

Il s'agit de ne pas s'aliéner l'auditeur-juge par un récit trop long. Comme le montre encore les incipit des histoires suivant celle de Philoclès, ce choix, quel qu'il soit, est déterminé par une stratégie rhétorique : il faut «persuader » par le discours, c'est-àdire, pour reprendre la définition de Furetière, «forcer quelqu'un à croire quelque chose ». Dans le cas d'un narrateur relatant sa propre histoire, ce choix est aussi lié à un tempérament. Pour l'un, les grandes douleurs sont muettes, pour l'autre, il faut qu'elles s'épanchent pour être persuasives. Aussi le prince Artibie, l'« Amant en deuil », fait-il le choix de la brièveté pour ne point s'infliger la souffrance de la remémoration :

Le souvenir des malheurs, est sans doute assez agreable, à ceux qui ne les souffrent plus : \& qui comme des gens échapez au naufrage, racontent les périls qu'ils ont évitez, n'estant plus en lieu, ny en estat de les pouvoir craindre. Mais le mal que je souffre estant un mal eternel, ou qui du moins ne finira qu'avec ma vie : il ne me seroit pas aisé d'avoir l'esprit assez libre, pour vous pouvoir raconter exactement, la naissance \& le progrés de ma passion [...]. Dispensez moy donc, je vous en conjure, de m'estendre sur tout ce qui ne sera point funeste: \& ne trouvez pas mauvais, que mon ame accoustumee à ne penser qu'à la mort, ne vous entretienne que de choses melancoliques : \& ne remplisse vostre imagination, que d'Urnes, de Cendres \& de Tombeaux. Je ne vous diray point ${ }^{30}[. .$.$] .$

Mais Léontidas est d'avis contraire :

Comme la douleur agit differemment, selon les divers temperamens de ceux qu'elle possede; qu'elle est tantôt muette, \& puis tantost eloquente : vous ne devez pas vous estonner si elle ne fait point en mon esprit, ce qu'elle a fait en celuy du prince Artibie, qui n'a pû s'estendre dans sa narration par l'excés de ses desplaisirs. Pour moy qui ne suis pas de ceux que la douleur fait taire, \& qui au contraire ne parle jamais tant, que lors que j'ay sujet de me pleindre, je n'en sçaurois user de cette sorte: \& je ne sçaurois, ce me semble, vous persuader en peu de paroles, la grandeur de mes souffrances. Je ne vous diray pourtant rien d'inutile si je le puis ${ }^{31}$ [...].

10 L'incipit du récit le plus court d'Artamene, l'« Histoire de Phylidas, et d'Anaxandride " (16 pages), est intéressant, parce qu'il rappelle la règle sans chercher à la justifier - la narratio judiciaire doit être brève -, signalant par là qu'elle est connue et admise de tous les auditeurs-lecteurs. C'est ce que fait comprendre, en effet, «il n'est pas à propos presentement » dans le passage suivant, qui renvoie au contexte du tribunal d'amour donc au modèle de la narratio : 
Comme il n'est pas à propos presentement, Madame, de faire un fort long recit, je ne m'amuseray point à vous dire ce qu'estoient les Peres et ceux dont j'ay à vous raconter l'Histoire : \& je me contenteray de vous assurer ${ }^{32}[. .$.$] .$

L'examen de cette histoire permet de dégager les caractéristiques du récit bref, par comparaison avec les récits plus développés. La nature de l'histoire racontée concourt à sa brièveté : nous avons ici affaire à une histoire galante, une histoire d'amour privée, sans enjeux politiques qui rendent nécessaire une mise en contexte historique et politique. Cette histoire, qui ne met en scène que trois personnages, le héros, l'héroïne et la rivale de l'héroïne qui sont associées à deux lieux, Mégare et Salamine, est peu chargée en péripéties ${ }^{33}$. Ces lieux ne sont que mentionnés et non décrits : on ne trouve aucune information géographique et rien sur les mœurs des peuples. Les personnages sont rapidement présentés et ne sont pas l'occasion d'un portrait physique et moral développé. On ne trouve pas non plus, autre genre bref souvent inséré dans le genre long qu'est le roman, de lettres reproduites comme en fac-similé : le contenu des missives échangées n'est qu'évoqué ${ }^{34}$. Le narrateur passe rapidement sur la rencontre des héros, la naissance et la progression de leur amour, en sorte que le récit est tôt consacré à la crise. Et surtout, on ne trouve qu'à deux reprises du discours rapporté directement, une réplique d'Anaxandride au narrateur d'environ une page, et une partie d'un dialogue entre ce même narrateur et le héros, d'environ deux pages et demie. Ces deux passages, et c'est là le point important, sont consacrés à l'exposition et à l'explication par les personnages des sentiments qu'ils ressentent : ce ne sont pas des discours pathétiques ${ }^{35}$.

11 Nous avons donc ici affaire à une narration très particulière : même les récits les plus courts contiennent abondamment $\mathrm{du}$ discours rapporté indirectement et, majoritairement, directement. Un tel choix de la part d'un narrateur ne peut donc que susciter les récriminations du public, que représente ici Eumétis : les amateurs de longs romans n'aiment pas la brièveté. Comme Mandane, l'héroïne principale, la maîtresse de Cyrus, ils préfèrent les narrations étendues. Celle-ci, alors qu'Orcame s'apprête à entamer la relation de l'« Histoire du Roy d'Assirie, d'Intapherne, d'Atergatis, \& de la Princesse de Bithinie », déclare :

La seule grace que je vous demande [...] est que vous ne fassiez pas comme ceux qui en faisant une narration, n'ont autre dessein que de dire beaucoup de choses en peu de paroles : car enfin il y a certains evenemens, où l'exageration est si agreable, \& mesme si necessaire pour les bien dire, que je ne puis souffrir ces avares de paroles, qui croyent avoir gagné beaucoup, quand ils ont espargné quelques silabes : c'est pourquoy ne renfermez point vostre esprit dans des bornes si estroites, \& ne songez à rien tant qu'à me dire tout ce que vous sçavez ${ }^{36}$.

C'est qu'il s'agit d'une histoire compliquée, qui joint intrigues politiques et amoureuses et met en scène de nombreux personnages. Sa narration sera difficile et requerra du talent: "J'ai tant de choses differentes à vous dire, que je doute si je pourray donner assez d'ordre à mon récit, pour faire qu'il ne vous ennuie pas ${ }^{37}$ ». Au terme d'une narration qui couvre 242 pages et qui passionne ses auditeurs, nous sommes invités à admirer le talent du narrateur. Les Scudéry font souvent le choix d'intrigues complexes, notamment parce que leur narration requiert un travail de construction important (dispositio), et que ce travail est pour eux la manifestation de leur art.

Cette narration pour être agréable doit émouvoir et, pour ce faire, recourir à l'exagération, figure de rhétorique assez connue pour être inventoriée dans le Dictionnaire de Furetière (1690) ${ }^{38}$ et dans celui de l'Académie (1694) qui donne d'« exagérer » cette définition : «Amplifier par discours, representer une chose encore 
plus grande, ou plus mauvaise, plus loüable, ou blasmable qu'elle n'est. Il a fort exaggeré cette victoire, l'importance de cette action, l'atrocité de ce crime. c'est un homme qui exaggere tousjours les choses». Il s'agit d'introduire du pathos dans la narration, et le moyen privilégié pour ce faire est le recours au discours, « expression faite de vive voix de ses pensées » (Dictionnaire de Furetière), " propos, assemblage de paroles pour expliquer ce que l'on pense " (Dictionnaire de l'Académie). Dans son sens premier, on le voit, le terme est lié à la parole orale, ce qui, dans le roman, amène à insérer en abondance du discours rapporté directement ou indirectement, monologue ou dialogue. C'est ainsi qu'il faut comprendre le commentaire liminaire de Léontidas cité plus haut: un narrateur qui veut persuader de la vertu des personnages dont il raconte l'histoire, de la force des sentiments qu'ils ressentent doit en passer par le discours rapporté, porte ouverte sur leur intériorité dont la connaissance est le meilleur moyen de persuader le lecteur de la possession effective par ces personnages des vertus que le narrateur leur reconnait, de l'expérience effective par ces personnages des joies et surtout des douleurs hors du commun qu'ils sont déclarés avoir ressenties. Il s'agit ainsi de faire en sorte que le personnage soit comme de la connaissance de l'auditeur-lecteur, et que ce dernier, condition sine qua non du plaisir du récit, s'intéresse ${ }^{39}$ au personnage qu'il met en scène ${ }^{40}$. Pour ce faire, les Scudéry considèrent qu'il faut un récit circonstancié et mettent en œuvre ce qu'ils ont théorisé dans la préface d'Ibrahim: il ne suffit pas de déclarer qu'un personnage est vertueux pour être convaincant, il faut en apporter des preuves qui sont non les actions mais les motivations des actions auxquelles les lecteurs accèdent par les paroles rapportées et les psychorécits qui les accompagnent ${ }^{41}$. Aussi, à Cyrus qui lui dit qu'il n'a guère le temps d'entendre un récit très circonstancié et qu'il ne dispose que de deux heures, le narrateur de la vie de Sapho répond-il :

comme je suis persuadé qu'il n'y a point une si grande Histoire qu'on ne puisse narrer en deux heures quand on le veut, je pense que je puis m'engager à vous dire celle de Sapho en ce temps là, quoy qu'il y ait beaucoup de longues conversations que je ne dois pas obmettre, si vous voulez que ces Princesses connaissent bien l'admirable Personne dont elles veulent savoir la vie ${ }^{42}$.

De plus, la vraisemblance des intrigues complexes et des caractères hors du commun requiert une narration détaillée donc longue. C'est ce que fait savoir le narrateur de l'« Histoire de Mazare » à ses auditeurs :

il faut que vous me faciez la grace de m'accorder une heure d'audiance [...] car si je vous aprenois d'abord ce que je vous diray à la fin de mon recit, vous en seriez peutestre si surpris que vous auriez peine à me croire: c'est pourquoy il importe extrémement que je dispose vostre esprit peu à peu, à se laisser persuader plusieurs choses fort surprenantes ${ }^{43}$.

Pour être crédible donc persuasif, ce narrateur réclame de pouvoir faire un récit minutieux, donc long. Il atteint pleinement son objectif, disculper Mazare, au terme d'un récit de 124 pages. La brièveté n'est donc pas la condition sine qua non de la persuasion ${ }^{44}$. La condition sine qua non est que le récit soit agréable à l'auditeur-lecteur. Et celui-ci ne juge jamais long un discours qui l'intéresse parce qu'il satisfait sa curiosité. Le critère de la brièveté est donc tout subjectif : rapidement, les lecteurs ont considéré que les longs romans gagneraient à être abrégés ${ }^{45}$, alors que les lecteurs contemporains de leur publication attendaient avec impatience la parution des diverses parties du roman qu'ils avaient entamé. 


\section{L'émancipation de la narration}

Deux situations méritent d'être observées de près. La première est celle de l'« Histoire de Belesis, d'Hermogene, de Cleodore, et de Leonise» qui s'inscrit explicitement dans un cadre judiciaire : Bélésis demande à Cyrus de juger « de la justice de [s]a cause » et de «l'injustice » de celle d'Hermogène ${ }^{46}$, et son histoire est longue (199 pages) parce que, dit-il, le différend qui les oppose « est de telle nature [...] qu'il est impossible qu'il puisse jamais estre bien entendu, à moins que de sçavoir toute la vie d'Hemogene \& toute la mienne ${ }^{47}$ ». À l'issue du récit, Cyrus et les autres auditeurs pensent qu'il ne sera guère difficile de réconcilier les partis en présence, pour diverses raisons qui sont rapportées succinctement au discours indirect ${ }^{48}$. Mais, pendant le temps qu'a duré la relation, les ennemis se sont réconciliés eux-mêmes : la narration n'a donc plus d'utilité pragmatique. Ne reste alors que son intérêt intrinsèque, non son intérêt par rapport à une cause. La seconde est l'« Histoire de Peranius, Prince de Phocee et de la princesse Cleonisbe ». La narration est faite par un envoyé de Péranius, Thrytème, pour obtenir que Cyrus reconnaisse son maître comme prince de Phocée. Mais Cyrus accorde d'emblée son soutien ${ }^{49}$. La narration n'a plus d'autre utilité que de satisfaire la « curiosité » de ses auditeurs, curiosité que le récit lui-même s'est ingénié à faire naître. Thrytème est venu accompagné de deux étrangers dont l'un se réjouit et l'autre s'afflige en entendant Cyrus. Tout cela attise la « curiosité $^{50}$ » de Cyrus et Mandane au point qu'ils réclament avec insistance que Thrytème la satisfasse. Mais, prévient celuici, ce sera une narration longue (257 pages) :

Ce que vous me demandez Seigneur [...] n'est pas une chose que je puisse vous apprendre en peu de mots, non plus que ce que la Princesse Mandane veut sçavoir ${ }^{51}$ [...].

C'est que la vie de Périanus est extraordinaire et que l'extraordinaire, pour être vraisemblable, doit être motivé ${ }^{52}$.

La longueur de la narration n'est plus alors conditionnée par l'utilité des éléments qui la compose pour la cause qu'elle sert, mais par l'intérêt qu'elle parvient à susciter. La narration alors en vient à fonctionner en autarcie : elle fait naître la curiosité et la satisfait, procurant ainsi au lecteur du plaisir. C'est, d'emblée, dans les longs romans la fonction du début in medias res ${ }^{53}$. De là la mention de la curiosité et de la nécessité de la satisfaire dans le passage qui précède l'insertion dans le récit principal d'une nouvelle histoire : la plupart des histoires insérées sont ainsi justifiées ${ }^{54}$. La narration peut alors être présentée comme un «divertissement ${ }^{55}$ ", une façon de passer «agréablement le temps ${ }^{56} »$.

\section{Conclusion}

Les Scudéry ont une pratique réflexive de l'écriture romanesque d'une finesse remarquable, que ce soit dans les préfaces de leurs romans ou au fil de passages métadiscursifs insérés dans leurs narrations fictionnelles. L'observation de ces remarques nous apprend que ce qui est en jeu pour eux dans le choix de la narration brève ou longue n'est rien moins que l'émancipation du récit de toute cause, au sens rhétorique du terme: le récit se développera ce qu'il faut pour que la curiosité du lecteur-auditeur soit satisfaite. 
l'intrigue première, et des intrigues purement galantes. Les imbroglios politiques des romans héroïques engagent des intrigues complexes donc longues à démêler, et la narration de celles-ci a généralement une visée pragmatique que le contexte du récit inséré précise. D'une façon ou d'une autre, elle importe au déroulement de l'action. A contrario, les intrigues qui ne sont qu'amoureuses, "galantes" pour reprendre un terme du temps, trouvent souvent leur raison d'être ailleurs, dans la satisfaction de la curiosité que des personnages ressentent envers d'autres personnages qu'ils veulent connaître en profondeur, dans l'intérêt pour ce que nous appelons désormais la psychologie $e^{57}$. Comme le cas de la très longue « Histoire de Sapho ", celui de l'« Histoire d'Artaxandre et de Telamire » au troisième livre de la huitième partie est à cet égard exemplaire. Mandane a repéré, dans une troupe d'étrangers qui l'a rejointe au cours du voyage qui la ramène chez le roi son père, une femme et un homme. Elle demande à sa confidente, Martésie de s'informer à leur sujet, ce qu'elle réussit à faire en obtenant qu'Érinice raconte l'histoire de ses amis Artaxandre et Télamire, qui est une histoire purement privée, relativement brève (123 pages) et qui ne sera jugée que sur le plaisir qu'elle suscite ${ }^{58}$.

17 Ces remarques nous apprennent aussi que la narration brève est un choix judicieux quand elle est au service d'une argumentation dont elle est un élément, comme dans le cadre de la narration judiciaire mais que, délivrée de ses visées rhétoriques traditionnelles, la narration peut se consacrer à l'analyse en profondeur de l'homme, qui ne peut se faire succinctement tant le caractère des hommes est complexe :

En effet il faut sçavoir distinguer tous les divers degrez de melancolie, \& d'enjoüement: \& ne se contenter pas de dire en general, c'estoit une Personne serieuse, ou une Personne enjoüée, comme il y a beaucoup de Gens qui font : car il est certain qu'il y a mille petites observations à faire, qui mettent une notable difference, entre des temperammens qui ne semblent pas opposez ${ }^{59}$.

Et la «Peinture fidele du cœur, \& de l'esprit de Sapho » court sur 280 pages, pour la plus grande satisfaction des auditeurs-lecteurs.

La force de persuasion de la narration n'est plus alors au service d'une cause, mais au service d'elle-même. Elle permet de mettre en place un univers fictionnel crédible et intéressant, un univers dans lequel va s'immerger avec plaisir le lecteur pour qui les personnages sont » comme de sa connaissance ». Mais il n'appartient qu'aux narrateurs talentueux de dévoiler les arcanes du cœur des hommes, et de rendre crédibles des histoires si insolites qu'on ne peut rien en deviner, comme le fait savoir Arbatane à Cyrus, alors qu'il s'apprête à lui raconter la fin des aventures d'Agladitas, une histoire dont Cyrus « admir[e] la bizarrerie et la nouveauté60 ${ }^{6}$, une fois qu'il l'a entendue.

Ce qui est aussi constaté dans ce roman, c'est qu' " on n'aime pas trop faire une longue narration devant beaucoup de monde ${ }^{61} »$ : la narration brève est adaptée au discours public, la narration longue au discours privé, au discours destiné à peu de destinataires voire à un seul. Mutatis mutandis, elle est adaptée à la narration scripturale, au roman. 


\section{NOTES}

1. Pour avoir une bonne idée de la structure de ce roman et de la longueur des narrations, voir

R. Godenne, Les Romans de mademoiselle de Scudéry, Genève, Droz, 1983.

2. Paris, A. Courbé, 1656 (Genève, Slatkine Reprints, 1972), partie II, livre 1 (II, 1), p. 3-4 : « [...] Chrisante fut le lendemain au matin au lever du Roy de Phrigie : pour luy apprendre par un récit moins estendu, que celuy du jour precedent, tout ce qu'il avoit desja raconté de la merveilleuse vie de son cher Maistre : à la reserve des choses dont ce Prince avoit esté le tesmoing. Mais comme ils jugerent qu'il estoit à propos de ne laisser pas Ciaxare sans qu'il y eust quelqu'un aupres de luy, qui peust l'empescher de prendre une resolution violente contre Artamene ; le Roy de Phrigie dit qu'il valoit mieux qu'il y allast [...]. Qu'ainsi il faloit que Chrisante achevast de luy dire en peu de mots le reste de la vie d'Artamene, dont il avoit veû la plus grande partie : \& qu'en suite il pourroit tout à loisir en aprendre toutes les particularitez, à ceux qui en avoient desja sçeu le commencement, d'une façon estenduë. Chrisante trouvant que ce Prince avoit raison, satisfit sa curiosité : \& le charma si puissamment par son recit, quoy que ce ne fust qu'un simple Abregé de la vie d'Aratmene; qu'il redoubla encore de beaucoup l'estime qu'il avoit pour luy [...] ». Toutes nos références renvoient à l'édition que nous venons de citer.

3. Voir p. 94-95 de la première partie, la discussion qui précède le récit de la vie d'Artamène.

4. Pour limiter notre relevé à la page 83 : «tesmoigner contre», "accusation», " complice», « criminel », « innocent », « accusent », « innocent ».

5. Ibid., VII, 2, p. 425 : "Philocles qui souhaitant, pour diverses raisons, que cette Reine ne demeurast pas dans la volonté qu'elle avoit, se resolut de dire à Cyrus, ce que presques nul autre que luy ne luy pouvoit dire : afin qu'entrant dans les sentimens qu'il souhaitoit qu'il eust, il pûst apres cela conseiller à Timochare, qui avoit quelque credit aupres de Cleobuline, de persuader cette Princesse, de ne s'opiniatrer pas dans son dessein. ». Voir aussi à l'issue de la narration, le propos adressé par Cyrus au narrateur, p. 544.

6. Ibid., IX, 2, p. 457. Les Scudéry font du roman d'Honoré d'Urfé un de leurs modèles (voir la préface de leur premier roman, Ibrahim ou l'illustre Bassa, 1641). Sur les tribunaux d'amour, notamment dans l'Astrée, voir F. Greiner, « La Juridiction des sentiments : tribunaux et cours d'amour dans le roman français de l'âge baroque ", dans Du Roman courtois au roman baroque, dir. E. Bury et F. Mora, Paris, Les Belles Lettres, 2004, p. 181-192. Un autre patron narratif, celui de l'histoire exemplaire, est toujours présent mais dans une moindre mesure que dans l'Astrée. Voir par exemple ibid., IX, 1, p. 49 cette ouverture de récit: «Lors que je considere, Seigneur, par quelles voyes les Dieux font arriver les evenemens les plus surprenans; \& quel est cét indissoluble enchainement des petites choses aux grandes, \& des grandes aux petites ; je ne puis que je n'admire leur conduite, \& que je n'avoüe que ce n'est point aux hommes à la vouloir penetrer.».

7. Ibid., III, 1, p. 58-62.

8. Ibid., p. 62.

9. Ibid., p. 63.

10. Ibid., VII, 3.

11. Ibid. : « accuser », p. 646 et 648 , « juges », p. 649.

12. Voir l'ouverture de son récit, p. 649.

13. Ibid., p. 750-751.

14. Ibid., IX, II, p. 383-399.

15. Ibid., p. 400-456.

16. Ibid., la confirmation occupe les pages 456 à 465 puis vient le jugement, p. 465 . Notons que là encore l'organisation du discours est stipulée : les deux narrateurs rapporteront chacun leur 
« Exemple » et diront ensuite leurs « raisons » (p. 382). Cette organisation est précisée à l'issue du premier exemple: l'auditrice-juge principale demande au premier narrateur de repousser l'exposé de ses raisons à l'issue de la narration du deuxième exemple, « afin que raisonnant apres esgalement sur tous les deux, la chose en fust mieux esclaircie» (p. 399).

17. Ibid., VI, 3, p. 584-719.

18. Ibid., VI, 569.

19. Ibid, 575-576.

20. Ibid., VII, 1, p. 71-77.

21. Ibid., VI, 2, p. 320.

22. Ibid., p. 23.

23. Ibid.

24. Ibid., p. 322.

25. Ibid., VII, 2, p. 427, incipit de l'« Histoire de Cleobuline reine de Corinthe »: « Encore que ce soit la coustume de quelqu'un, de reprendre les choses d'assez loin ; \& de ne parler gueres moins des Peres de ceux de qui ils ont à narrer l'Histoire, que de ceux mesmes qui ont le plus d'interest à l'advanture qu'ils ont à dire, je n'en useray pourtant pas ainsi. ».

26. Ibid., p. 428 « Je sçay bien encore, Seigneur, que c'est l'usage, afin qu'on ne soit pas surpris du merite extraordinaire d'une Personne, de dire comme elle a esté eslevée, comment elle a apris toutes choses qu'elle sçait ; \& de commencer l'Histoire de sa vie dés le Berçeau. ».

27. Les «Histoires des Amants infortunez» sont composées de quatre narrations, celle de l'«Amant absent »: 83 pages; celle de l'«Amant non-aimé »: 63 pages; celle de l'« Amant en deuil »: 21 pages; et celle de l'« Amant jaloux » : 78 pages. Le tribunal où s'opposent Mnésiphile et Chersias contient un premier exemple de 16 pages, l'« Histoire de Phylidas et d'Anaxandrine » et un autre de 56 pages, l'« Histoire d'Aglatonice, et d'Iphicrate ». La narration de l'« Histoire de Trasyle ", qui doit disculper le personnage de l'accusation d'inconstance, est une configuration différente; il n'y a qu'un seul récit, qui est plus long : 100 pages. La plupart des récits seconds occupent entre 150 et 250 pages. Une unique exception : la dernière histoire insérée (chacun des 30 livres composant le roman contient une histoire insérée, à part le premier livre). Cette dernière histoire prend place à l'acmé de la crise, ce qui explique qu'elle ne peut qu'être brève, au moment où Cyrus est cru mort alors qu'il a été fait prisonnier incognito par Méliante. Mais il a été reconnu par Méréonte, qui convainc son ami Méliante de ne pas révéler l'identité du conquérant et d'aider ce dernier à fuir. Cela ne peut se faire que le lendemain. Dans l'entretemps, « comme ce Prince sçavoit bien que rien n'est plus obligeant que de tesmoigner d'avoir quelque curiosité pour ce qui regarde la fortune de ses Amis» (X, 3, p.652), il engage Méréonte à lui raconter son histoire. Là encore, le choix d'une narration brève est expliqué dans l'incipit du récit (p. 655).

28. Ibid., IX, 2, p. 400.

29. Ibid., III, 1, p. 155 (incipit de l'histoire de « L'Amant non aimé »).

30. Ibid., p. 223-224 (incipit de l'histoire de « L'Amant en deuil »).

31. Ibid., p. 248 (incipit de l'histoire de « L'Amant jaloux »).

32. Ibid., IX, 2, p. 383.

33. À Mégare, Phylidas aime Anaxandride qu'il doit épouser mais cette certitude le détache de sa maîtresse en sorte qu'il devient amoureux à Salamine de Timoxène, ce qui provoque le désamour d'Anaxandride. Mais depuis que cette dernière ne l'aime plus et le maltraite, il s'est repris à l'aimer.

34. Ibid., IX, 2, p. 386.

35. Sur les discours pathétiques dans les fictions narratives $\mathrm{du} \mathrm{XVII}^{\mathrm{e}}$ siècle, voir la thèse d'Adrienne Petit, Le Discours romanesque des passions. Rhétorique et poétique des passions dans la fiction narrative en prose du XVII siècle, soutenue en 2016 à l'université de Paris Sorbonne sous la direction de Delphine Denis. 
36. Artamene, VIII, 1, p. 52.

37. Ibid., p. 52.

38. « EXAGERATION. Figure de Rhetorique par laquelle on augmente ou on diminuë les choses, on dit plus ou moins qu'il n'y en a.».

39. Dictionnaire de Furetière : «INTERESSER, se dit aussi en Morale de l'émotion des passions. Un bon Orateur doit Interesser les Juges, les Esmouvoir à la colere, à la compassion. on s'interesse dans les spectacles; dans des representations fabuleuses, quand l'Auteur sçait bien Esmouvoir les passions. ».

40. Artamene, VIII, 3, p. 629 : "si on ne s'interesse un peu à la Fortune de ceux de qui on entend raconter l'Histoire, on n'y sçauroit prendre plaisir », déclare une narratrice avant d'engager sa narration.

41. Ibrahim, éd. A. Arrigoni, Fasano-Paris, Schena-PUPS, 2003, p. 83 : «Certainement il n'est rien de plus important, dans cette espece de composition, que d'imprimer fortement l'Idée, ou pour mieux dire, l'image des Heros en l'esprit du Lecteur : mais en façon qu'ils soyent comme de sa conoissance car c'est ce qui l'interesse en leurs avantures, \& de là que vient son plaisir. Or pour les faire connoistre parfaitement, il ne suffit pas de dire combien de fois ils ont fait naufrage, \& combien de fois ils ont rencontré des voleurs : mais il faut juger par leurs discours, quelles sont leurs inclinations: autrement l'on est en droit de dire à ces Heros muets ce beau mot de l'antiquité, PARLE AFIN QUE JE TE VOYE. ». Cf. Quintilien, Institution oratoire, IV, 2, 64 : « euidentia in narratione, quantum ego intellego, est quidem magna uirtus, cum quid ueri non dicendum, sed quodammodo etiam ostendendum est " (« quant à l'évidence dans la narration, pour autant que je comprenne le terme, c'est, à vrai dire, une grande qualité, quand il faut non pas tant dire que montrer du doigt, en quelque sorte, ce qui est vrai. », trad. J. Cousin, Paris, Les Belles Lettres, t. III, 1976, p. 56).

42. Artamene, X, 2, p. 326.

43. Ibid., VIII, 2 p. 369.

44. Voir à ce sujet l'article de Marie Formarier sur la conception de la brièveté chez Cicéron à partir du De oratore, conception qui est aussi celle des Scudéry, «La narratio chez Cicéron doit-elle être brève pour persuader? », Interférences (http://journals.openedition.org/interferences/6007), $\mathrm{n}^{\circ} 10,2018$.

45. Pour le roman d'Honoré d'Urfé : en 1678, Astrée. Premiere partie (C. Barbin), en 1712, La Nouvelle Astrée (N. Pepie), en 1733, L'Astrée de M. d'Urfé, Pastorale allégorique (P. Witte et Didot). Pour le roman de Desmarets: en 1724, Ariane (La Compagnie des Libraires). Pour Cléopâtre de La Calprenède: dès 1667, Abrégé de la Cléôpatre (T. Jolly) et, en 1789, Cléopâtre, roman historique (Maradan). Pour Cassandre du même La Calprenède : en 1752, un abrégé (P. Dusmenil et Vve Pissot) ; une réédition en 1731 (Montalant) et une autre, à Liège, en 1750 : Le Roman de Cassandre (E. Kintz). Enfin, pour Faramond, toujours de La Calprenède : un abrégé paraît en 1753 (Bauche).

46. Artamene, V, 3, p. 522.

47. Ibid., p. 521.

48. Ibid., p. 723.

49. Ibid, VIII, 2, p. 334 : « Ha Seigneur [...], je n'ay plus rien à vous demander ! car puis que vous reconnoissez en presence de ces Estrangers, Peranius pour Prince, \& pour Prince de Phocée, vous faites tout ce que j'avois ordre de vous supplier de faire ".

50. Le mot est employé deux fois page 334 et une fois à la page suivante.

51. Ibid, p. 335.

52. G. Genette, « Vraisemblance et motivation », Communications, $n^{\circ} 11,1968$, p. 5-21.

53. Voir T. Cave, "Suspendere animos: pour une histoire de la notion de suspens", dans Les Commentaires et la naissance de la critique littéraire, France/Italie (XIV-XVI siècles), dir. G. MathieuCastellani et M. Plaisance, Paris, Klincksieck, 1990, p. 211-218, et « Suspense and the Pre-History of the Novel », Revue de littérature comparée, n² 4, 1996, p. 507-516. 
54. Pour nous limiter aux quatre premiers tomes du roman, des 11 histoires secondes qu'ils contiennent, 8 sont ainsi justifiées. Le mot «curiosité » intervient dans le passage précédent l'ouverture de la narration dans 7 cas, et dans le dernier, c'est l'expression «brûler du désir de savoir» (Artamene, I, 2, p. 96).

55. Ibid, VIII, 3, p. 629.

56. Ibid., X, 2, p. 357.

57. Cyrus demande à Démocédès de faire un récit minutieux de la vie de Sapho parce qu' «il n'y a sans doute rien qui attache davantage l'esprit de ceux qui doivent escouter une Histoire, que de leur faire bien connoistre la Personne qui y a interest: \& que de la leur representer si parfaitement, qu'ils puissent presques s'imaginer qu'ils la connoissent par eux mesmes» (p. 326-327). Pour ce faire, lui répond Démocédès, il ne faut pas se contenter d'une description superficielle, physique, mais sonder le cœur et l'esprit de son personnage, en sorte d'en dévoiler la singularité, et la chose est difficile : «il n'est pas aussi aisé de faire une Peinture fidelle, du cœur, de l'esprit, \& de toutes les inclinations d'une Personne, que de son visage : puis qu'il est vray qu'à moins que d'avoir un certain esprit de discernement, qui sçait trouver de la difference entre les choses qui paroissent semblables, à ceux qui ne les examinent pas bien, il n'est pas aisé de faire une Peinture bien ressemblante. » (IX, 2, p. 326-327). L'Histoire insérée est l'une des plus longues, qui court sur 280 pages.

58. Ibid., VIII, 3, p. 754 : «Comme Erinice eut achevé son recit, \& que Doralise se preparoit à la remercier du plaisir qu'elle avoit eu à l'escouter, Amaldée entra [...]».

59. Ibid., X, 2, p. 327. L'histoire qui va être livrée à la suite de ce commentaire est celle de Sapho (personnage à clef : mademoiselle de Scudéry); elle est destinée à faire connaître précisément qui est cette femme illustre. Elle n'est que très lâchement rattachée à l'intrigue principale. Le contexte insiste sur la curiosité pour Sapho (trois occurrences du mot aux pages 324 et 325).

60. Ibid., IV, 2, p. 384.

61. Ibid., VIII, 2, p. 346, constat que fait Cyrus avant que Thrytème n'engage une narration qui va être longue de 257 pages, le lecteur, on l'a vu, ayant été averti de la longueur p. 335. Que quelques personnages (voire un seul) se mettent à l'écart, souvent dans un cabinet, pour entendre une histoire, est fréquent dans les longs romans.

\section{AUTEUR}

\section{MARIE-GABRIELLE LALLEMAND}

Université Caen Normandie 